\title{
Towards Statistical Reasoning in Description Logics over Finite Domains
}

\author{
Rafael Peñaloza ${ }^{1}$ and Nico Potyka ${ }^{2}$ \\ 1 KRDB Research Centre, Free University of Bozen-Bolzano, Italy \\ rafael.penaloza@unibz.it \\ ${ }^{2}$ University of Osnabrück, Germany \\ npotyka@uni-osnabrueck.de
}

\begin{abstract}
We present a probabilistic extension of the description logic $\mathcal{A L C}$ for reasoning about statistical knowledge. We consider conditional statements over proportions of the domain and are interested in the probabilistic-logical consequences of these proportions. After introducing some general reasoning problems and analyzing their properties, we present first algorithms and complexity results for reasoning in some fragments of Statistical $\mathcal{A L C}$.
\end{abstract}

\section{Introduction}

Probabilistic logics enrich classical logics with probabilities in order to incorporate uncertainty. In [5], probabilistic logics have been classified into three types that differ in the way how they handle probabilities. Type 1 logics enrich classical interpretations with probability distributions over the domain and are well suited for reasoning about statistical probabilities. This includes proportional statements like " $2 \%$ of the population suffer from a particular disease." Type 2 logics consider probability distributions over possible worlds and are better suited for expressing subjective probabilities or degrees of belief. For instance, a medical doctor might say that she is $90 \%$ sure about her diagnosis. Type 3 logics combine type 1 and type 2 logics allow to reason about both kinds of uncertainty.

One basic desiderata of probabilistic logics is that they generalize a classical logic. That is, the probabilistic interpretation of formulas with probability 1 should agree with the classical interpretation. However, given that first-order logic is undecidable, a probabilistic first-order logic that satisfies our basic desiderata will necessarily be undecidable. In order to overcome the problem, we can, for instance, restrict to Herbrand interpretations over a fixed domain $[2,9,13]$ or consider decidable fragments like description logics $[3,8,10]$.

Probabilistic type 2 extensions of description logics have been previously studied in [11]. In the unpublished appendix of this work, a type 1 extension of $\mathcal{A L C}$ is presented along with a proof sketch for EXPTIME-completeness of the corresponding satisfiability problem. This type 1 extension enriches classical interpretations with probability distributions over the domain as suggested in [5]. We consider a similar, but more restrictive setting here. We are interested in an $\mathcal{A L C}$ extension that allows statistical reasoning. However, we do not impose a probability distribution over the domain. Instead, 
we are only interested in reasoning about the proportions of a population satisfying some given properties. For instance, given statistical information about the relative frequency of certain symptoms, diseases and the relative frequency of symptoms given diseases, one can ask the relative frequency of a disease given a particular combination of symptoms. Therefore, we consider only classical $\mathcal{A L C}$ interpretations with finite domains and are interested in the relative proportions that are true in these interpretations.

Hence, interpretations in our framework can be regarded as a subset of the interpretations in [11], namely those with finite domains and a uniform probability distribution over the domain. These interpretations are indeed sufficient for our purpose. In particular, by considering strictly less interpretations, we may be able to derive tighter answer intervals for some queries. Our approach bears some resemblance to the random world approach from [4]. However, the authors in [4] consider possible worlds with a fixed domain size $N$ and are interested in the limit of proportions as $N$ goes to infinity. We are interested in all finite possible worlds that satisfy certain proportions and ask what statistical statements must be true in all these worlds.

We begin by introducing Statistical $\mathcal{A L C}$ in Section 2 together with three relevant reasoning problems. Namely, the Satisfiability Problem, the 1-Entailment problem and the p-Entailment problem. In Section 3, we will then discuss some logical properties of Statistical $\mathcal{A L C}$. In Section 4 and 5, we present first computational results for fragments of Statistical $\mathcal{A L C}$. We had to omit several proofs in order to meet space restrictions. All proofs can be found in the full version of the paper [16].

\section{Statistical $\mathcal{A L C}$}

We start by revisiting the classical description logic $\mathcal{A L C}$. Given two disjoint sets $\mathrm{N}_{\mathrm{C}}$ of concept names and $\mathrm{N}_{\mathrm{R}}$ of role names, $\mathcal{A L C}$ concepts are built using the grammar rule $C::=\top|A| \neg C|C \sqcap C| \exists r$. $C$, where $A \in \mathrm{N}_{\mathrm{C}}$ and $r \in \mathrm{N}_{\mathrm{R}}$. One can express disjunction, universal quantification and subsumption through the usual logical equivalences like $C_{1} \sqcup C_{2} \equiv \neg\left(\neg C_{1} \sqcap \neg C_{2}\right)$. For the semantics, we focus on finite interpretations. An $\mathcal{A L C}$ interpretation $\mathcal{I}=\left(\Delta^{\mathcal{I}},{ }^{\mathcal{I}}\right)$ consist of a non-empty, finite domain $\Delta^{\mathcal{I}}$ and an interpretation function ${ }^{\mathcal{I}}$ that maps concept names $A \in \mathrm{N}_{\mathrm{C}}$ to sets $A^{\mathcal{I}} \subseteq \Delta^{\mathcal{I}}$ and roles names $r \in \mathrm{N}_{\mathrm{R}}$ to binary relations $r^{\mathcal{I}} \subseteq \Delta^{\mathcal{I}} \times \Delta^{\mathcal{I}}$. Two $\mathcal{A L C}$ concepts $C_{1}, C_{2}$ are equivalent $\left(C_{1} \equiv C_{2}\right)$ iff $C_{1}^{\mathcal{I}}=C_{2}^{\mathcal{I}}$ for all interpretations $\mathcal{I}$.

Here, we consider a probabilistic extension of $\mathcal{A L C}$. Statistical $\mathcal{A L C}$ knowledge bases consist of probabilistic conditionals that are built up over $\mathcal{A L C}$ concepts.

Definition 1 (Conditionals, Statistical KB). A probabilistic $\mathcal{A L C}$ conditional is an expression of the form $(C \mid D)[\ell, u]$, where $C, D$ are $\mathcal{A L C}$ concepts and $\ell, u \in \mathbb{Q}$ are rational numbers such that $0 \leq \ell \leq u \leq 1$. A statistical $\mathcal{A L C}$ knowledge base $(K B)$ is a set $\mathcal{K}$ of probabilistic $\mathcal{A L C}$ conditionals.

For brevity, we usually call probabilistic $\mathcal{A L C}$ conditionals simply conditionals.

Example 2. Let $\mathcal{K}_{f l u}=\{(\exists$ has.fever $\mid \exists$ has.flu $)[0.9,0.95],(\exists$ has.flu $\mid \top)[0.01,0.03]\}$. $\mathcal{K}_{f l u}$ states that 90 to 95 percent of patients who have the flu have fever, and that only 1 to 3 percent of patients have the flu. 
Intuitively, a conditional $(C \mid D)[\ell, u]$ expresses that the relative proportion of elements of $D$ that also belong to $C$ is between $\ell$ and $u$. In order to make this more precise, consider a finite $\mathcal{A L C}$ interpretation $\mathcal{I}$, and an $\mathcal{A L C}$ concept $X$. We denote the cardinality of $X^{\mathcal{I}}$ by $[X]^{\mathcal{I}}$, that is, $[X]^{\mathcal{I}}:=\left|X^{\mathcal{I}}\right|$. The interpretation $\mathcal{I}$ satisfies $(C \mid D)[\ell, u]$, written as $\mathcal{I} \models(C \mid D)[\ell, u]$, iff either $[D]^{\mathcal{I}}=0$ or

$$
\frac{[C \sqcap D]^{\mathcal{I}}}{[D]^{\mathcal{I}}} \in[\ell, u] .
$$

$\mathcal{I}$ satisfies a statistical $\mathcal{A L C}$ knowledge base $\mathcal{K}$ iff it satisfies all conditionals in $\mathcal{K}$. In this case, we call $\mathcal{I}$ a model of $\mathcal{K}$ and write $\mathcal{I} \models \mathcal{K}$. We denote the set of all models of $\mathcal{K}$ by $\operatorname{Mod}(\mathcal{K})$. As usual, $\mathcal{K}$ is consistent if $\operatorname{Mod}(\mathcal{K}) \neq \emptyset$ and inconsistent otherwise. We call two knowledge bases $\mathcal{K}_{1}, \mathcal{K}_{2}$ equivalent and write $\mathcal{K}_{1} \equiv \mathcal{K}_{2}$ iff $\operatorname{Mod}\left(\mathcal{K}_{1}\right)=\operatorname{Mod}\left(\mathcal{K}_{2}\right)$.

Example 3. Consider again the $\mathrm{KB} \mathcal{K}_{f l u}$ from Example 2. Let $\mathcal{I}$ be an interpretation with 1000 individuals. 10 of these have the flu and 9 have both the flu and fever. Then $\mathcal{I} \in \operatorname{Mod}\left(\mathcal{K}_{f l u}\right)$.

In classical $\mathcal{A L C}$, knowledge bases are defined by a set of general concept inclusions (GCIs) $C \sqsubseteq D$ that express that $C$ is a subconcept of $D$. An interpretation $\mathcal{I}$ satisfies $C \sqsubseteq D$ iff $C^{\mathcal{I}} \subseteq D^{\mathcal{I}}$. As shown next, GCIs can be seen as a special kind of conditionals, and hence statistical $\mathcal{A L C}$ KBs are a generalization of classical $\mathcal{A L C}$ KBs.

Proposition 4. For all statistical $\mathcal{A L C}$ interpretations $\mathcal{I}$, we have $\mathcal{I} \models C \sqsubseteq D$ iff $\mathcal{I} \models(D \mid C)[1,1]$.

Proof. If $\mathcal{I} \models C \sqsubseteq D$ then $C^{\mathcal{I}} \subseteq D^{\mathcal{I}}$ and $C^{\mathcal{I}} \cap D^{\mathcal{I}}=C^{\mathcal{I}}$. If $C^{\mathcal{I}}=\emptyset$, we have $[C]^{\mathcal{I}}=0$. Otherwise $\frac{[C \sqcap D]^{\mathcal{I}}}{[C]^{\mathcal{I}}}=1$. Hence, $\mathcal{I}=(D \mid C)[1,1]$.

Conversely, assume $\mathcal{I}=(D \mid C)[1,1]$. If $[C]^{\mathcal{I}}=0$, then $C^{\mathcal{I}}=\emptyset$ and $\mathcal{I}=C \sqsubseteq D$. Otherwise, $\frac{[C \sqcap D]^{\mathcal{I}}}{[C]^{\mathcal{I}}}=1$, that is, $[C \sqcap D]^{\mathcal{I}}=[C]^{\mathcal{I}}$. If there was a $d \in C^{\mathcal{I}} \backslash D^{\mathcal{I}}$, we had $[C \sqcap D]^{\mathcal{I}}<[C]^{\mathcal{I}}$, hence, we have $C^{\mathcal{I}} \subseteq D^{\mathcal{I}}$ and $\mathcal{I}=C \sqsubseteq D$.

Given a statistical $\mathcal{A L C}$ knowledge base $\mathcal{K}$, the first problem that we are interested in is deciding consistency of $\mathcal{K}$. We define the satisfiability problem for statistical $\mathcal{A L C}$ knowledge bases as usual.

Satisfiability Problem: Given a knowledge base $\mathcal{K}$, decide whether $\operatorname{Mod}(\mathcal{K}) \neq \emptyset$.

Example 5. Consider again the knowledge base $\mathcal{K}_{f l u}$ from Example 2. The conditional ( $\exists$ has.flu $\mid T)[0.01,0.03]$ implies that $[\exists \text { has.flu }]^{\mathcal{I}} \geq 0.01$ for all models $\mathcal{I} \in \operatorname{Mod}\left(\mathcal{K}_{f l u}\right)$. (ᄏhas.fever $\mid \exists$ has.flu) $[0.9,0.95]$ implies $[\exists \text { has.fever } \sqcap \exists \text { has.flu }]^{\mathcal{I}} \geq 0.9[\exists \text { has.flu }]^{\mathcal{I}}$. Therefore, $[\exists \text { has.fever }]^{\mathcal{I}} \geq[\exists \text { has.fever } \sqcap \exists \text { has.flu }]^{\mathcal{I}} \geq 0.9[\exists \text { has.flu }]^{\mathcal{I}} \geq 0.009$. Hence, adding the conditional ( $\exists$ has.fever $\mid \top)[0,0.005]\}$ renders $\mathcal{K}_{f u}$ inconsistent.

If $\mathcal{K}$ is consistent, we are interested in deriving (implicit) probabilistic conclusions. We can think of different reasoning problems in this context. First, we can define an entailment relation analogously to logical entailment. Then, the probabilistic conditional $(C \mid D)[\ell, u]$ is an l-consequence of the $\mathrm{KB} \mathcal{K}$ iff $\operatorname{Mod}(\mathcal{K}) \subseteq \operatorname{Mod}(\{(C \mid D)[\ell, u]\})$. In this case, we write $\mathcal{K} \models_{l}(C \mid D)[\ell, u]$. In the context of type 2 probabilistic conditionals, this entailment relation has also been called just logical consequence [9]. 
l-Entailment Problem: Given a knowledge base $\mathcal{K}$ and a conditional $(C \mid D)[\ell, u]$, decide whether $\mathcal{K} \models_{l}(C \mid D)[\ell, u]$.

Example 6. Consider again the $\mathrm{KB} \mathcal{K}_{f u}$ from Example 2. As explained in Example 5, $[\exists \text { has.fever }]^{\mathcal{I}} \geq 0.009$ holds for all models $\mathcal{I} \in \operatorname{Mod}(\mathcal{K})$. Therefore, it follows that $\mathcal{K}_{f l u} \models_{l}$ ( $\exists$ has.fever $\left.\mid \top\right)[0.009,1]$. That is, our statistical information suggests that at least 9 out of 1,000 of our patients have fever.

Example 7. Consider a domain with birds (B), penguins $(\mathrm{P})$ and flying animals $(\mathrm{F})$. We let $\mathcal{K}_{\text {birds }}=\{(B \mid \top)[0.5,0.6],(F \mid B)[0.85,0.9],(F \mid P)[0,0]\}$. Note that the conditional $(F \mid B)[0.85,0.9]$ is actually equivalent to $(\neg F \mid B)[0.1,0.15]$. Furthermore, for all $\mathcal{I} \in \operatorname{Mod}\left(\mathcal{K}_{\text {birds }}\right),(F \mid P)[0,0]$ implies $[P \sqcap F]^{\mathcal{I}}=0$. Therefore, we have $[P \sqcap B]^{\mathcal{I}}=[B \sqcap P \sqcap F]^{\mathcal{I}}+[B \sqcap P \sqcap \neg F]^{\mathcal{I}} \leq 0+[B \sqcap \neg F]^{\mathcal{I}} \leq 0.15[B]^{\mathcal{I}}$. Hence, $\mathcal{K}_{\text {birds }}=_{l}(P \mid B)[0,0.15]$. That is, our statistical information suggests that at most 15 out of 100 birds in our population are penguins.

As usual, the satisfiability problem can be reduced to the l-entailment problem.

Proposition 8. $\mathcal{K}$ is inconsistent iff $\mathcal{K} \models_{l}(\top \mid \top)[0,0]$.

Proof. If $\mathcal{K}$ is inconsistent, then $\operatorname{Mod}(\mathcal{K})=\emptyset$ and so $\mathcal{K} \models_{l}(T \mid T)[0,0]$.

Conversely, assume $\mathcal{K} \models_{l}(T \mid T)[0,0]$. We have $[T]^{\mathcal{I}}>0$ and $\frac{[T \cap T]^{\mathcal{I}}}{[T]^{\mathcal{I}}}=1$ for all interpretations $\mathcal{I}$. Hence, $\operatorname{Mod}(\{(T \mid T)[0,0]\})=\emptyset$ and since $\mathcal{K} \models_{l}(T \mid T)[0,0]$, we $\operatorname{must}$ have $\operatorname{Mod}(\mathcal{K})=\emptyset$ as well.

Often, we do not want to check whether a specific conditional is entailed, but rather deduce tight probabilistic bounds for a statement. This problem is often referred to as the probabilistic entailment problem in other probabilistic logics, see $[6,9,13]$ for instance. Consider a query of the form $(C \mid D)$, where $C, D$ are $\mathcal{A L C}$ concepts. We define the p-Entailment problem similar to the probabilistic entailment problem for type 2 probabilistic logics.

p-Entailment Problem: Given knowledge base $\mathcal{K}$ and a query $(C \mid D)$, find minimal and maximal solutions of the optimization problems

$$
\begin{array}{lc}
\inf _{\mathcal{I} \in \operatorname{Mod}(\mathcal{K})} / \sup _{\mathcal{I} \in \operatorname{Mod}(\mathcal{K})} & \frac{[C \sqcap D]^{\mathcal{I}}}{[D]^{\mathcal{I}}} \\
\text { subject to } & {[D]^{\mathcal{I}}>0}
\end{array}
$$

Since the objective function $\frac{[C \sqcap D]^{\mathcal{I}}}{[D]^{\mathcal{I}}}$ is bounded from below by 0 and from above by 1 , the infimum $m$ and the maximum $M$ are well-defined whenever there is a model $\mathcal{I} \in \operatorname{Mod}(\mathcal{K})$ such that $[D]^{\mathcal{I}}>0$. In this case, we say that $\mathcal{K}$ p-entails $(C \mid D)[m, M]$ and write $\mathcal{K} \models_{p}(C \mid D)[m, M]$. In the context of type 2 probabilistic conditionals, this entailment relation has also been called tight logical consequence [9]. If $[D]^{\mathcal{I}}=0$ for all $\mathcal{I} \in \operatorname{Mod}(\mathcal{K})$, the p-Entailment problem is infeasible, that is, there exists no solution. 
Example 9. In Example 7, we found that $\mathcal{K}_{\text {birds }} \models_{l}(P \mid B)[0,0.15]$. This bound is actually tight. Since 0 is always a lower bound and we showed that 0.15 is an upper bound, it suffices to give examples of interpretations that take these bounds. For the lower bound, let $\mathcal{I}_{0}$ be an interpretation with 200 individuals. 100 of these individuals are birds and 85 are birds that can fly. There are no penguins. Then $\mathcal{I}_{0}$ is a model of $\mathcal{K}_{\text {birds }}$ with $[B]^{\mathcal{I}_{0}}>0$ that satisfies $(P \mid B)[0,0]$. Construct $\mathcal{I}_{1}$ from $\mathcal{I}_{0}$ by letting the 15 non-flying birds be penguins. Then $\mathcal{I}_{1}$ is another model of $\mathcal{K}_{\text {birds }}$ and $\mathcal{I}_{1}$ satisfies $(P \mid B)[0.15,0.15]$. Hence, we also have $\mathcal{K}_{\text {birds }} \models_{p}(P \mid B)[0,0.15]$.

If $\mathcal{K} \models_{p}(C \mid D)[m, M]$, one might ask whether the values between $m$ and $M$ are actually taken by some model of $\mathcal{K}$ or whether there can be large gaps in between. For the probabilistic entailment problem for type 2 logics, we can show that the models of $\mathcal{K}$ do indeed yield a dense interval by noting that each convex combination of models is a model and applying the Intermediate Value Theorem from Real Analysis. However, in our framework, we do not consider probability distributions over possible worlds, but the worlds themselves, which are discrete in nature. We therefore cannot apply the same tools here. However, for each two models that yield different probabilities for a query, we can find another model that takes the probability in the middle of these probabilities.

Lemma 10 (Bisection Lemma). Let $C, D$ be two arbitrary $\mathcal{A L C}$ concepts. If there exist $\mathcal{I}_{0}, \mathcal{I}_{1} \in \operatorname{Mod}(\mathcal{K})$ such that $r_{0}=\frac{[C \sqcap D]^{\mathcal{I}_{0}}}{[D]^{\mathcal{I}_{0}}}<\frac{[C \sqcap D]^{\mathcal{I}_{1}}}{[D]^{\mathcal{I}_{1}}}=r_{1}$, then there is an $\mathcal{I}_{0.5} \in \operatorname{Mod}(\mathcal{K})$ such that $\frac{[C \sqcap D]^{\mathcal{I}_{0.5}}}{[D]^{\mathcal{I}_{0.5}}}=\frac{r_{0}+r_{1}}{2}$.

We can now show that for each value between the lower and upper bound given by p-entailment, we can find a model that gives a probability arbitrarily close to this value.

Proposition 11 (Intermediate Values). Let $\mathcal{K} \models_{p}(C \mid D)[m, M]$. Then for every $x \in(m, M)$ (where $(m, M)$ denotes the open interval between $m$ and $M$ ) and for all $\epsilon>0$, there is a $\mathcal{I}_{x, \epsilon} \in \operatorname{Mod}(\mathcal{K})$ such that $\left|\frac{[C \sqcap D]^{\mathcal{I}_{x, \epsilon}}}{[D]^{\mathcal{I}_{x, \epsilon}}}-x\right|<\epsilon$.

\section{Logical Properties}

We now discuss some logical properties of Statistical $\mathcal{A} \mathcal{L C}$. We already noted that Statistical $\mathcal{A L C}$ generalizes classical $\mathcal{A L C}$ in Proposition 4. Furthermore, p-entailment yields a tight and dense (Proposition 11) answer interval for all queries whose condition can be satisfied by models of the knowledge base. Let us also note that statistical $\mathcal{A L C}$ is language invariant. That is, increasing the language by adding new concept or role names does not change the semantics of $\mathcal{A L C}$. This can be seen immediately by observing that the interpretation of conditionals in (1) depends only on the concept and role names that appear in the conditional.

Statistical $\mathcal{A L C}$ is also representation invariant in the sense that for all concepts $C_{1}, D_{1}$ and $C_{2}, D_{2}$, if $C_{1} \equiv C_{2}$ and $D_{1} \equiv D_{2}$ then $\left(C_{1} \mid D_{1}\right)[l, u] \equiv\left(C_{2} \mid D_{2}\right)[l, u]$. Hence, changing the syntactic representation of conditionals does not change their semantics. In particular, entailment results are independent of such changes.

Both 1- and p-entailment satisfy the following independence property: whether or not $\mathcal{K} \models_{l}(C \mid D)[\ell, u]\left(\mathcal{K} \models_{p}(C \mid D)[m, M]\right)$ depends only on the conditionals 
in $\mathcal{K}$ that are connected with the query. This may simplify answering the query by reducing the size of the KB. In order to make this more precise, we need some additional definitions. For an arbitrary $\mathcal{A L C}$ concept $C, \operatorname{Sig}(C)$ denotes the set of all concept and role names appearing in $C$. The conditionals $\left(C_{1} \mid D_{1}\right)\left[\ell_{1}, u_{1}\right]$ and $\left(C_{2} \mid D_{2}\right)\left[\ell_{2}, u_{2}\right]$ are directly connected (written $\left.\left(C_{1} \mid D_{1}\right)\left[\ell_{1}, u_{1}\right] \rightleftharpoons\left(C_{2} \mid D_{2}\right)\left[\ell_{2}, u_{2}\right]\right)$ if and only if $\left(\operatorname{Sig}\left(C_{1}\right) \cup \operatorname{Sig}\left(D_{1}\right)\right) \cap\left(\operatorname{Sig}\left(C_{2}\right) \cup \operatorname{Sig}\left(D_{2}\right)\right) \neq \emptyset$. That is, two conditionals are directly connected iff they share concept or role names. Let $\rightleftharpoons^{*}$ denote the transitive closure of $\rightleftharpoons$. We say that $\left(C_{1} \mid D_{1}\right)\left[\ell_{1}, u_{1}\right]$ and $\left(C_{2} \mid D_{2}\right)\left[\ell_{2}, u_{2}\right]$ are connected iff $\left(C_{1} \mid D_{1}\right)\left[\ell_{1}, u_{1}\right] \rightleftharpoons{ }^{*}\left(C_{2} \mid D_{2}\right)\left[\ell_{2}, u_{2}\right]$. The restriction of $\mathcal{K}$ to conditionals connected to $(C \mid D)[\ell, u]$ is the set $\left\{\kappa \in \mathcal{K} \mid \kappa \rightleftharpoons^{*}(C \mid D)[\ell, u]\right\}$. Using an analogous definition for queries (qualitative conditionals) $\left(C_{1} \mid D_{1}\right)$ and $\left(C_{2} \mid D_{2}\right)$, we get the following result.

Proposition 12 (Independence). If $\mathcal{K}$ is consistent, we have

$$
\begin{aligned}
& \text { 1. } \mathcal{K} \models_{l}(C \mid D)[\ell, u] \text { iff }\left\{\kappa \in \mathcal{K} \mid \kappa \rightleftharpoons^{*}(C \mid D)[\ell, u]\right\} \models_{l}(C \mid D)[\ell, u] \text {. } \\
& \text { 2. } \mathcal{K} \models_{p}(C \mid D)[m, M] \text { iff }\left\{\kappa \in \mathcal{K} \mid \kappa \rightleftharpoons^{*}(C \mid D)\right\} \models_{p}(C \mid D)[m, M] .
\end{aligned}
$$

Another interesting property of probabilistic logics is continuity. Intuitively, continuity states that minor changes in the knowledge base do not yield major changes in the derived probabilities. However, as demonstrated by Courtney and Paris, this condition is too strong when reasoning with the maximum entropy model of the knowledge base [14, p. 90]. The same problem arises for the probabilistic entailment problem [17, Example 4]. While these logics considered subjective probabilities, the same problem occurs in our setting for statistical probabilities as we demonstrate now.

Example 13. Consider the knowledge base

$$
\mathcal{K}=\{(B \mid A)[0.4,0.5],(C \mid A)[0.5,0.6],(B \mid C)[1,1],(C \mid B)[1,1]\} .
$$

The interpretation $\mathcal{I}=\left(\{a, b\},{ }^{\mathcal{I}}\right)$ with $A^{\mathcal{I}}=\{a, b\}, B^{\mathcal{I}}=C^{\mathcal{I}}=\{b\}$ is a model of $\mathcal{K}$, i.e., $\mathcal{K}$ is consistent. In particular, since $A$ is interpreted by the whole domain of $\mathcal{I}$ we know that

$$
\mathcal{K}={ }_{p}(A \mid \top)[m, 1]
$$

for some $m \in[0,1]$. As explained in Proposition 4, deterministic conditionals correspond to concept inclusions and so $(B \mid C)[1,1]$ and $(C \mid B)[1,1]$ imply that $B^{\mathcal{I}^{\prime}}=C^{\mathcal{I}^{\prime}}$ for all models $\mathcal{I}^{\prime}$ of $\mathcal{K}$. Therefore, $\frac{\left[B \sqcap A \mathcal{I}^{\mathcal{I}^{\prime}}\right.}{[A]^{I^{\prime}}}=\frac{[C \sqcap A]^{\mathcal{I}^{\prime}}}{[A]^{\mathcal{I}^{\prime}}}$. Let $\mathcal{K}^{\prime}$ denote the knowledge base that is obtained from $\mathcal{K}$ by decreasing the upper bound of the first conditional in $\mathcal{K}$ by an arbitrarily small $\epsilon>0$. That is,

$$
\mathcal{K}^{\prime}=\{(B \mid A)[0.4,0.5-\epsilon],(C \mid A)[0.5,0.6],(B \mid C)[1,1],(C \mid B)[1,1]\} .
$$

Then the only way to satisfy the first two conditionals in $\mathcal{K}^{\prime}$ is by interpreting $A$ by the empty set. Indeed, the interpretation $\mathcal{I}_{\emptyset}$ that interprets all concept names by the empty set is a model of $\mathcal{K}^{\prime}$. So $\mathcal{K}^{\prime}$ is consistent and

$$
\mathcal{K}^{\prime} \models_{p}(A \mid \top)[0,0] .
$$


Hence, a minor change in the probabilities in the knowledge base can yield a severe change in the entailed probabilities. This means that the p-entailment relation that we consider here is not continuous in this way either.

As an alternative to this strong notion of continuity, Paris proposed to measure the difference between KBs by the Blaschke distance between their models. Blaschke continuity says that if KBs are close with respect to the Blaschke distance, the entailed probabilities are close. Blaschke continuity is satisfied by some probabilistic logics under maximum entropy and probabilistic entailment $[14,17]$. In $[14,17]$, probabilistic interpretations are probability distributions over a finite number of classical interpretations and the distance between two interpretations is the distance between the corresponding probability vectors. We cannot apply this definition here because we interpret conditionals by means of classical interpretations. It is not at all clear what a reasonable definition for the distance between two classical interpretations is. We leave the search for a reasonable topology on the space of classical interpretations for future work.

\section{Statistical $\mathcal{E L}$}

Proposition 4 and the fact that reasoning in $\mathcal{A L C}$ is EXPTIME-complete, show that our reasoning problems are EXPTIME-hard. However, we did not find any upper bounds on the complexity of reasoning in $\mathcal{A L C}$ so far. We will therefore focus on some fragments of $\mathcal{A L C}$ now.

To begin with, we will focus on the sublogic $\mathcal{E L}$ [1] of $\mathcal{A L C}$ that does not allow for negation and universal quantification. Formally, $\mathcal{E} \mathcal{L}$ concepts are constructed by the grammar rule $C::=A|\top| C \sqcap C \mid \exists r$. $C$, where $A \in N_{C}$ and $r \in N_{R}$. A statistical $\mathcal{E L}$ $K B$ is a statistical $\mathcal{A L C} \mathrm{KB}$ where conditionals are restricted to $\mathcal{E L}$ concepts. Notice that, due to the upper bounds in conditionals, statistical $\mathcal{E L}$ KBs are capable of expressing some weak variants of negations. For instance, a statement $(C \mid \top)[\ell, u]$ with $u<1$ restricts every model $\mathcal{I}=\left(\Delta^{\mathcal{I}},{ }^{\mathcal{I}}\right)$ to contain at least one element $\delta \in \Delta^{\mathcal{I}} \backslash C^{\mathcal{I}}$. Thus, contrary to classical $\mathcal{E} \mathcal{L}$, statistical $\mathcal{E L}$ KBs may be inconsistent.

Example 14. Consider the $\mathrm{KB} \mathcal{K}_{1}=\left(\emptyset, \mathcal{C}_{1}\right)$, where

$$
\mathcal{C}_{1}=\{(A \mid \top)[0,0.2],(A \mid \top)[0.3,1]\} .
$$

Since $\top^{\mathcal{I}}=\Delta^{\mathcal{I}} \neq \emptyset$, every model $\mathcal{I}=\left(\Delta^{\mathcal{I}},{ }^{\mathcal{I}}\right)$ of $\mathcal{K}_{1}$ must satisfy

$$
[A]^{\mathcal{I}} \leq 0.2[\top]^{\mathcal{I}}<0.3[\top]^{\mathcal{I}} \leq[A]^{\mathcal{I}}
$$

which is clearly a contradiction. Thus, $\mathcal{K}_{1}$ is inconsistent.

More interestingly, though, it is possible to simulate valuations over a finite set of propositional formulas wit the help of conditional statements. Thus, the satisfiability problem is at least NP-hard even for Statistical $\mathcal{E L}$.

Theorem 15. The satisfiability problem for Statistical $\mathcal{E L}$ is NP-hard. 
On the other hand, consistency can be decided in non-deterministic exponential time, through a reduction to integer programming. Before describing the reduction in detail, we introduce a few simplifications.

Recall from Proposition 4 that a conditionals of the form $(D \mid C)[1,1]$ is equivalent to the classical GCI $C \sqsubseteq D$. Thus, in the following we will often express statistical $\mathcal{E L}$ KBs as pairs $\mathcal{K}=(\mathcal{T}, \mathcal{C})$, where $\mathcal{T}$ is a classical TBox (i.e., a finite set of GCIs), and $\mathcal{C}$ is a set of conditionals. A statistical $\mathcal{E L} \mathrm{KB} \mathcal{K}=(\mathcal{T}, \mathcal{C})$ is said to be in normal form if all the GCIs in $\mathcal{T}$ are of the form

$$
A_{1} \sqcap A_{2} \sqsubseteq B, \quad A \sqsubseteq \exists r . B, \quad \exists r . A \sqsubseteq B
$$

and all its conditionals are of the form

$$
(A \mid B)[\ell, u]
$$

where $A, B \in N_{C} \cup\{\top\}$, and $r \in N_{R}$. Informally, a KB is in normal form if at most one constructor is used in any GCI, and all conditionals are atomic (i.e., between concept names). Every KB can be transformed to an equivalent one (w.r.t. the original signature) in linear time using the normalization rules from [1], and introducing new concept names for complex concepts appearing in conditionals. More precisely, we replace any conditional of the form $(C \mid D)[\ell, u]$ with the statement $(A \mid B)[\ell, u]$, where $A, B$ are two fresh concept names, and extend the TBox with the axioms $A \equiv C$, and $B \equiv D$.

The main idea behind our consistency algorithm is to partition the finite domain of a model into the different types that they define, and use integer programming to verify that all the logical and conditional constraints are satisfied. Let $N_{C}(\mathcal{K})$ denote the set of all concept names appearing in the KB $\mathcal{K}$. We call any subset $\theta \subseteq N_{C}(\mathcal{K})$ a type for $\mathcal{K}$. Intuitively, such a type $\theta$ represents all the elements of the domain that are interpreted to belong to all concept names $A \in \theta$ and no concept name $A \notin \theta$. We denote as $\Theta(\mathcal{K})$ the set of all types of $\mathcal{K}$. To simplify the presentation, in the following we treat $T$ as a concept name that belongs to all types.

Given a statistical $\mathcal{E} \mathcal{L} \mathrm{KB} \mathcal{K}=(\mathcal{T}, \mathcal{C})$ in normal form, we consider an integer variable $x_{\theta}$ for every type $\theta \in \Theta(\mathcal{K})$. These variables will express the number of domain elements that belong to the corresponding type. In addition, $x_{\top}$ will be used to represent the total size of the domain. We build a system of linear inequalities over these variables as follows. First, we require that all variables have a value at least 0 , and that the sizes of all types add exactly the size of the domain.

$$
\begin{aligned}
\sum_{\theta \in \Theta(\mathcal{K})} x_{\theta} & =x_{\top} \\
0 & \leq x_{\theta} \quad \text { for all } \theta \in \Theta(\mathcal{K})
\end{aligned}
$$

Then, we ensure that all the conditional statements from the KB are satisfied by adding, for each statement $(A \mid B)[\ell, u] \in \mathcal{C}$ the constraint

$$
\ell \cdot \sum_{B \in \theta} x_{\theta} \leq \sum_{A, B \in \theta} x_{\theta} \leq u \cdot \sum_{B \in \theta} x_{\theta},
$$


Finally, we must ensure that the types satisfy all the logical constraints introduced by the TBox. The GCI $A_{1} \sqcap A_{2} \sqsubseteq B$ states that every element that belongs to both $A_{1}$ and $A_{2}$ must also belong to $B$. This means that types containing $A_{1}, A_{2}$ but excluding $B$ should not be populated. We thus introduce the inequality

$$
x_{\theta}=0 \quad \text { if } A_{1} \sqcap A_{2} \sqsubseteq B \in \mathcal{T}, A_{1}, A_{2} \in \theta \text {, and } B \notin \theta
$$

Dealing with existential restrictions requires checking different alternatives, which we solve by creating different linear programs. The GCI $A \sqsubseteq \exists r . B$ implies that, whenever there exists an element in $A$, there must also exist at least one element in $B$. Thus, to satisfy this axiom, either $A$ should be empty (i.e., $\sum_{A \in \theta} x_{\theta}=0$ ), or $\sum_{B \in \theta} x_{\theta} \geq 1$. Hence, for every existential restriction of the form $A \sqsubseteq \exists$ r. $B$, we define the set

$$
\mathcal{E}_{A, B}:=\left\{\sum_{A \in \theta} x_{\theta}=0, \sum_{B \in \theta} x_{\theta} \geq 1\right\}
$$

To deal with GCIs of the form $\exists r . A \sqsubseteq B$, we follow a similar approach, together with the ideas of the completion algorithm for classical $\mathcal{E L}$. For every pair of existential restrictions $A \sqsubseteq \exists$ r. $B, \exists r . C \sqsubseteq D$, we define the set

$$
\mathcal{F}_{A, B, C, D}:=\left\{\sum_{A \in \theta, D \notin \theta} x_{\theta}=0, \sum_{B \in \theta, C \notin \theta} x_{\theta} \geq 1\right\}
$$

Intuitively, $\sum_{A \in \theta, D \notin \theta} x_{\theta} \geq 1$ whenever there exists an element that belongs to $A$ but not to $D$. If this is the case, and the GCIs $A \sqsubseteq \exists r . B, \exists r . C \sqsubseteq D$ belong to the TBox $\mathcal{T}$, then there must exist some element that belongs to $B$ but not to $C$.

We call the hitting sets of

$$
\left\{\mathcal{E}_{A, B} \mid A \sqsubseteq \exists r . B \in \mathcal{T}\right\} \cup\left\{\mathcal{F}_{A, B, C, D} \mid A \sqsubseteq \exists r . B, \exists r . C \sqsubseteq D \in \mathcal{T}\right\}
$$

choices for $\mathcal{T}$. A program for $\mathcal{K}$ is an integer program containing all the inequalities (2)(5) and a choice for $\mathcal{T}$. Then we get the following result.

Lemma 16. $\mathcal{K}$ is consistent iff there exists a program for $\mathcal{K}$ that is satisfiable.

Proof. The "only if" direction is straight-forward since the inequalities are sound w.r.t. the semantics of statistical KBs. We focus on the "if" direction only.

Given a solution of the integer program, we construct an interpretation $\mathcal{I}=\left(\Delta,{ }^{\mathcal{I}}\right)$ as follows. We create a domain $\Delta$ with $x_{\top}$ elements, and partition it such that for every type $\theta \in \Theta(\mathcal{K})$, there is a class $[[\theta]]$ containing exactly $x_{\theta}$ elements. For every nonempty class, select a representative element $\delta_{\theta} \in[[\theta]]$.

The interpretation function.${ }^{\mathcal{I}}$ maps every concept name $A$ to the set

$$
A^{\mathcal{I}}:=\bigcup_{A \in \theta}[[\theta]]
$$

Given a non-empty class $[[\theta]]$ such that $A \in \theta$ and $A \sqsubseteq \exists r . B \in \mathcal{T}$, let $\tau$ be a type such that $B \in \tau, x_{\tau}>0$, and for every $\exists r . C \sqsubseteq D \in \mathcal{T}$, if $D \notin \theta$, then $C \notin \tau$. Notice 
that such a $\tau$ must exist because the solution must satisfy at least one restriction in each $\mathcal{F}_{A, B, C, D}$. We define $r_{A, B}^{\theta}:=\theta \times\left\{\delta_{\tau}\right\}$ and set

$$
r^{\mathcal{I}}:=\bigcup_{A \in \theta, A \sqsubseteq \exists r . B \in \mathcal{T}} r_{A, B}^{\theta} .
$$

It remains to be shown that $\mathcal{I}$ is a model of $\mathcal{K}$.

Notice that for two concept names $A, B$, it holds that $(A \sqcap B)^{\mathcal{I}}=\bigcup_{A, B \in \theta}[[\theta]]$ and hence $[A \sqcap B]^{\mathcal{I}} \mid=\sum_{A, B \in \theta} x_{\theta}$. Given a conditional statement $(A \mid B)[\ell, u] \in \mathcal{C}$, since the solution must satisfy the inequality (4), it holds that

$$
\ell \cdot[B]^{\mathcal{I}} \leq[A \sqcap B]^{\mathcal{I}} \leq u \cdot[B]^{\mathcal{I}} .
$$

For a GCI $A_{1} \sqcap A_{2} \sqsubseteq B \in \mathcal{T}$, by the inequality (5) it follows that for every type $\theta$ containing both $A_{1}, A_{2}$, but not $B$, [[ $\left.[\theta]\right]=\emptyset$. Hence $A_{1}^{\mathcal{I}} \cap A_{2}^{\mathcal{I}} \subseteq B^{\mathcal{I}}$. For every $A \sqsubseteq \exists r . B \in \mathcal{T}$, and every $\gamma \in \Delta$, if $\gamma \in A^{\mathcal{I}}$ then by construction there is an element $\gamma^{\prime}$ such that $\left(\gamma, \gamma^{\prime}\right) \in r^{\mathcal{I}}$.

Finally, if $\left(\gamma, \gamma^{\prime}\right) \in r^{\mathcal{I}}$, then by construction there exists a type $\theta$ and an axiom $A \sqsubseteq \exists r . B \in \mathcal{T}$ such that $\gamma \in[[\theta]]$ and $\gamma^{\prime}=\delta_{\tau}$. Then, for every GCI $\exists r . C \sqsubseteq D \in \mathcal{T}$, $\gamma^{\prime} \in C^{\mathcal{I}}$ implies $C \in \tau$ and hence $D \in \theta$ which means that $\gamma \in D^{\mathcal{I}}$.

Notice that the construction produces exponentially many integer programs, each of which uses exponentially many variables, measured on the size of the KB. Since satisfiability of integer linear programs is decidable in non-deterministic polynomial time on the size of the program, we obtain a non-deterministic exponential time upper bound for deciding consistency of statistical $\mathcal{E L ~ K B s}$.

Theorem 17. Consistency of statistical $\mathcal{E L ~ K B s ~ i s ~ i n ~ N E X P T I M E . ~}$

\section{Reasoning with Open Minded KBs}

In order to regain tractability, we now further restrict statistical $\mathcal{E L}$ KBs by disallowing upper bounds in the conditional statements. We call such knowledge bases open minded.

Definition 18 (Open Minded KBs). A statistical $\mathcal{E} K K B \mathcal{K}=(\mathcal{T}, \mathcal{C})$ is open minded iff all the conditional statements $(C \mid D)[\ell, u] \in \mathcal{C}$ are such that $u=1$.

For the scope of this section, we consider only open minded KBs. The first obvious consequence of restricting to this class of $\mathrm{KBs}$ is that negations cannot be simulated. In fact, every open minded $\mathrm{KB}$ is consistent and, as in classical $\mathcal{E L}$, can be satisfied in a simple universal model.

Theorem 19. Every open minded KB is consistent.

Proof. Consider the interpretation $\mathcal{I}=\left(\{\delta\},{ }^{\mathcal{I}}\right)$ where the interpretation function maps every concept name $A$ to $A^{\mathcal{I}}:=\{\delta\}$ and every role name $r$ to $r^{\mathcal{I}}:=\{(\delta, \delta)\}$. It is easy to see that this interpretation is such that $C^{\mathcal{I}}=\{\delta\}$ holds for every $\mathcal{E} \mathcal{L}$ concept $C$. Hence, $\mathcal{I}$ satisfies all $\mathcal{E} \mathcal{L}$ GCIs and in addition $[C \sqcap D]^{\mathcal{I}}=[C]^{\mathcal{I}}=1$ which implies that all conditionals are also satisfied. 
Table 1. Rules for deciding $m$-necessity

\begin{tabular}{llrl}
\hline $\mathbf{C}_{1}$ & if $\left\{\left(X, A_{1}\right),\left(X, A_{2}\right)\right\} \subseteq \mathcal{S}$ and $A_{1} \sqcap A_{2} \sqsubseteq B \in \mathcal{T}$ & then add $(X, B)$ to $\mathcal{S}$ \\
$\mathbf{C}_{2}$ & if $(X, A) \in \mathcal{S}$ & and $A \sqsubseteq \exists r . B \in \mathcal{T}$ & then add $(A, r, B)$ to $\mathcal{S}$ \\
$\mathbf{C}_{3}$ & if $\{(X, r, Y),(Y, A)\} \subseteq \mathcal{S}$ and $\exists r . A \sqsubseteq B \in \mathcal{T}$ & then add $(X, B)$ to $\mathcal{S}$ \\
\hline $\mathbf{L}_{1}$ & if & $(A \mid B)[\ell, 1] \in \mathcal{C}$ & then $\mathcal{L}(A) \leftarrow \ell \cdot \mathcal{L}(B)$ \\
$\mathbf{L}_{2}$ & if & $A_{1} \sqcap A_{2} \sqsubseteq B \in \mathcal{T}$ & then $\mathcal{L}(B) \leftarrow \mathcal{L}\left(A_{1}\right)+\mathcal{L}\left(A_{2}\right)-1$ \\
$\mathbf{L}_{3}$ & if $(B, A) \in \mathcal{S}$ & & then $\mathcal{L}(A) \leftarrow \mathcal{L}(B)$ \\
\hline
\end{tabular}

Recall that, intuitively, conditionals specify that a proportion of the population satisfies some given properties. One interesting special case of $p$-entailment is the question how likely it is to observe an individual that belongs to a given concept.

Definition 20. Let $\mathcal{K}$ be an open minded $K B, C$ a concept, and $m \in[0,1]$. $C$ is $m$-necessary in $\mathcal{K}$ if $\mathcal{K}$ p-entails $(C \mid \top)[m, 1]$. The problem of $m$-necessity consists in deciding whether $C$ is $m$-necessary in $\mathcal{K}$.

We show that this problem can be solved in polynomial time. As in the previous section, we assume that the $\mathrm{KB}$ is in normal form and additionally, that all conditional statements $(A \mid B)[\ell, 1] \in \mathcal{C}$ are such that $\ell<1$. This latter assumption is made w.l.o.g. since the conditional statement $(A \mid B)[1,1]$ can be equivalently replaced by the GCI $B \sqsubseteq A$ (see Proposition 4). Moreover, checking $m$-necessity of a complex concept $C$ w.r.t. the $\mathrm{KB}(\mathcal{T}, \mathcal{C})$ is equivalent to deciding $m$-necessity of a new concept name $A$ w.r.t. the $\mathrm{KB}(\mathcal{T} \cup\{A \equiv C\}, \mathcal{C})$. Thus, in the following we consider w.l.o.g. only the problem of deciding $m$-necessity of a concept name w.r.t. to a KB in normal form.

Our algorithm extends the completion algorithm for classification of $\mathcal{E L}$ TBoxes to in addition keep track of the lower bounds of necessity for all relevant concept names. The algorithm keeps as data structure a set $\mathcal{S}$ of tuples of the form $(A, B)$ and $(A, r, B)$ for $A, B \in N_{C} \cup\{\top\}$. These intuitively express that the TBox $\mathcal{T}$ entails the subsumptions $A \sqsubseteq B$ and $A \sqsubseteq \exists$ r.B. respectively. Additionally, we keep a function $\mathcal{L}$ that maps every element $A \in N_{C} \cup\{\top\}$ to a number $\mathcal{L}(A) \in[0,1]$. Intuitively, $\mathcal{L}(A)=n$ expresses that $\mathcal{K}$ p-entails $(A \mid \top)[n, 1]$.

The algorithm initializes the structures $\mathcal{S}$ and $\mathcal{L}$ as

$$
\begin{aligned}
\mathcal{S} & :=\left\{(A, A),(A, \top) \mid A \in N_{C}(\mathcal{K}) \cup\{\top\}\right\} \\
\mathcal{L}(A) & := \begin{cases}0 & \text { if } A \in N_{C}(\mathcal{K}) \\
1 & \text { if } A=\top .\end{cases}
\end{aligned}
$$

These structures are then updated using the rules from Table 1. In each case, a rule is only applied if its execution extends the available knowledge; that is, if either $\mathcal{S}$ is extended to include one more tuple, or a lower bound in $\mathcal{L}$ is increased. In the latter case, only the larger value is kept through the function $\mathcal{L}$.

The first three rules in Table 1 are the standard completion rules for classical $\mathcal{E L}$. The remaining rules update the lower bounds for the likelihood of all relevant concept names, taking into account their logical relationship, as explained next. 
Rule $\mathbf{L}_{1}$ applies the obvious inference associated to conditional statements: from all the individuals that belong to $B,(A \mid B)[\ell, 1]$ states that at least $100 \ell \%$ belong also to $A$. Thus, assuming that $\mathcal{L}(B)$ is the lowest proportion of elements in $B$ possible, the proportion of elements in $A$ must be at least $\ell \cdot \mathcal{L}(B) . \mathbf{L}_{3}$ expresses that if every element of $B$ must also belong to $A$, then there must be at least as many elements in $A$ as there are in $B$. Finally, $\mathbf{L}_{2}$ deals with the fact that two concepts that are proportionally large must necessarily overlap. For example, if $60 \%$ of all individuals belong to $A$ and $50 \%$ belong to $B$, then at least $10 \%$ must belong to both $A$ and $B$; otherwise, together they would cover more than the whole domain.

The algorithm executes all the rules until saturation; that is, until no rule is applicable. Once it is saturated, we can decide $m$-necessity from the function $\mathcal{L}$ as follows: $A$ is $m$-necessary iff $m \leq \mathcal{L}(A)$. Before showing the correctness of this algorithm, we show an important property.

Notice that the likelihood information from $\mathcal{L}$ is never transferred through roles. The reason for this is that an existential restriction $\exists r . B$ only guarantee the existence of one element belonging to the concept $B$. Proportionally, the number of elements that belong to $B$ tends to 0 .

Example 21. Consider the $\mathrm{KB}(\{\top \sqsubseteq \exists r . A\}, \emptyset)$. For any $n \in \mathbb{N}$, construct the interpretation $\mathcal{I}_{n}:=\left(\{0, \ldots, n\},{ }^{\mathcal{I}_{n}}\right)$, where $A^{\mathcal{I}_{n}}=\{0\}$ and $r^{\mathcal{I}_{n}}=\{(k, 0) \mid 0 \leq k \leq n\}$. It is easy to see that $\mathcal{I}_{n}$ is a model of the KB and $[A]^{\mathcal{I}_{n}} /[T]^{I_{n}}<1 / n$. Thus, the best lower bound for $m$-necessity of $A$ is 0 , as correctly given by the algorithm.

Theorem 22 (correctness). Let $\mathcal{L}$ be the function obtained by the application of the rules until saturation and $A_{0} \in N_{C}$. Then $A_{0}$ is m-necessary iff $m \leq \mathcal{L}(A)$.

Proof (sketch). It is easy to see that all the rules are sound, which proves the "if" direction. For the converse direction, we consider a finite domain $\Delta$ and an interpretation ${ }^{\mathcal{I}}$ of the concept names such that $[A]^{\mathcal{I}} /|\Delta|=\mathcal{L}(A)$ and the post-conditions of the rules $\mathbf{L}_{1}-\mathbf{L}_{3}$ are satisfied. Such interpretation can be obtained recursively by considering the last rule application that updated $\mathcal{L}(A)$. Assume w.l.o.g. that the domain is large enough so that $c /|\Delta|<m-\mathcal{L}\left(A_{0}\right)$, where $c$ is the number of concept names appearing in $\mathcal{K}$. It is easy to see that this interpretation satisfies all conditional statements and the GCIs $A_{1} \sqcap A_{2} \sqsubseteq B \in \mathcal{T}$. For every concept name $A$, create a new domain element $\delta_{A}$ and extend the interpretation $\mathcal{I}$ such that $\delta_{A} \in B$ iff $(A, B) \in \mathcal{S}$. Given a role name $r$, we define $r^{\mathcal{I}}:=\left\{\left(\gamma, \delta_{B}\right) \mid A \sqsubseteq \exists r . B, \gamma \in A^{\mathcal{I}}\right\}$. Then, this interpretation satisfies the KB $\mathcal{K}$, and $\left[A_{0}\right]^{\mathcal{I}} /|\Delta| \leq \mathcal{L}\left(A_{0}\right)+c /|\Delta|<m$.

Thus, the algorithm can correctly decide $m$-necessity of a given concept name. It remains only to be shown that the process terminates after polynomially many rule applications. To guarantee this, we impose an ordering in the rule applications. First, we apply all the classical rules $\mathbf{C}_{\mathbf{1}}-\mathbf{C}_{3}$, and only when no such rules are applicable, we update the function $\mathcal{L}$ through the rules $\mathbf{L}_{1}-\mathbf{L}_{3}$. In this case, the rule that will update to the largest possible value is applied first. It is known that only polynomially many classical rules (on the size of $\mathcal{T}$ ) can be applied [1]. Deciding which bound rule to apply next requires polynomial time on the number of concept names in $\mathcal{K}$. Moreover, since the largest update is applied first, the value of $\mathcal{L}(A)$ is changed at most once for every 
concept name $A$. Hence, only linearly many rules are applied. Overall, this means that the algorithm terminates after polynomially many rule applications, which yields the following result.

Theorem 23. Deciding m-necessity is in $\mathrm{P}$.

\section{Related Work}

Over the years, various probabilistic extensions of description logics have been investigated, see, for instance, $[3,7,8,10,12,15,18]$. The one that is closest to our approach is the type 1 extension of $\mathcal{A L C}$ proposed in the appendix of [11]. Briefly, [11] introduces probabilistic constraints of the form $P(C \mid D) \leq p, P(C \mid D)=p, P(C \mid D) \geq p$ for $\mathcal{A L C}$ concepts $C, D$. These correspond to the conditionals $(C \mid D)[0, p],(C \mid D)[p, p]$, $(C \mid D)[p, 1]$, respectively. Conversely, each conditional can be rewritten as such a probabilistic constraint. However, there is a subtle but fundamental difference in the semantics. While the definition in [11] allows for probability distributions over arbitrary domains, we do not consider uncertainty over the domain. This comes down to allowing only finite domains and only the uniform distribution over this domain; that is, our approach further restricts the class of models of a KB. One fundamental difference between the two approaches is that Proposition 4 does not hold in [11]: the reason is that the conditional $(C \mid D)[1,1]$ can be satisfied by an interpretation $\mathcal{I}$ that contains an element $x \in(C \sqcap \neg D)^{\mathcal{I}}$, where $x$ has probability 0 .

This difference is the main reason why the EXPTIME algorithm proposed by Lutz and Schröder cannot be transferred to our setting. It does not suffice to consider the satisfiable types independently, but other implicit subsumption relations may depend on the conditionals only.

Example 24. Consider the statistical $\mathcal{E L} \mathrm{KB} \mathcal{K}=(\mathcal{T}, \mathcal{C})$ with

$$
\begin{aligned}
\mathcal{T} & :=\{\top \sqsubseteq \exists r . A, \quad \exists r . B \sqsubseteq C\} \\
\mathcal{C} & :=\{(B \mid \top)[0.5,1], \quad(A \mid B)[0.5,1], \quad(A \mid \top)[0,0.25]\}
\end{aligned}
$$

From $\mathcal{C}$ it follows that every element of $A$ must also belong to $B$, and hence every domain element must be an element of $C$. However, $\neg C$ defines a satisfiable type (w.r.t. $\mathcal{T}$ ) which will be interpreted as non-empty in the model generated by the approach in [11].

\section{Conclusions}

We have introduced Statistical $\mathcal{A L C}$, a new probabilistic extension of the description logic $\mathcal{A L C}$ for statistical reasoning. We analyzed the basic properties of this logic and introduced some reasoning problems that we are interested in. As a first step towards effective reasoning in Statistical $\mathcal{A L C}$, we focused on $\mathcal{E L}$, a well-known sublogic of $\mathcal{A L C}$ that, in its classical form, allows for polynomial-time reasoning. We showed that upper bounds in conditional constraints make the satisfiability problem in statistical 
$\mathcal{E L}$ NP-hard and gave an NEXPTIME algorithm to decide satisfiability. We showed that tractability can be regained by disallowing strict upper bounds in the conditional statements.

We are going to provide more algorithms and a more complete picture of the complexity of reasoning for Statistical $\mathcal{A L C}$ and its fragments in future work. A combination of integer programming and the inclusion-exclusion principle may be fruitful to design first algorithms for reasoning in full Statistical $\mathcal{A L C}$.

\section{References}

1. Baader, F., Brandt, S., Lutz, C.: Pushing the $\mathcal{E} \mathcal{L}$ envelope. In: Kaelbling, L.P., Saffiotti, A. (eds.) Proc. of IJCAI'05. pp. 364-369. Morgan-Kaufmann (2005)

2. Beierle, C., Kern-Isberner, G., Finthammer, M., Potyka, N.: Extending and completing probabilistic knowledge and beliefs without bias. KI-Künstliche Intelligenz 29(3), 255-262 (2015)

3. Ceylan, İ.İ., Peñaloza, R.: The bayesian ontology language $\mathcal{B E} \mathcal{L}$. J. Autom. Reasoning 58(1), 67-95 (2017)

4. Grove, A.J., Halpern, J.Y., Koller, D.: Random worlds and maximum entropy. In: Logic in Computer Science, 1992. LICS'92., Proceedings of the Seventh Annual IEEE Symposium on. pp. 22-33. IEEE (1992)

5. Halpern, J.Y.: An analysis of first-order logics of probability. Artificial intelligence 46(3), 311-350 (1990)

6. Hansen, P., Jaumard, B.: Probabilistic satisfiability. In: Kohlas, J., Moral, S. (eds.) Handbook of Defeasible Reasoning and Uncertainty Management Systems, vol. 5, pp. 321-367. Springer Netherlands (2000)

7. Klinov, P., Parsia, B.: Pronto: A practical probabilistic description logic reasoner. In: Uncertainty Reasoning for the Semantic Web II, pp. 59-79. Springer (2013)

8. Koller, D., Levy, A., Pfeffer, A.: P-classic: a tractable probablistic description logic. AAAI/IAAI 1997, 390-397 (1997)

9. Lukasiewicz, T.: Probabilistic logic programming with conditional constraints. ACM Trans. Comput. Logic 2(3), 289-339 (Jul 2001)

10. Lukasiewicz, T., Straccia, U.: Managing uncertainty and vagueness in description logics for the semantic web. JWS 6(4), 291-308 (2008)

11. Lutz, C., Schröder, L.: Probabilistic description logics for subjective uncertainty. In: Proc. KR 2010. AAAI Press (2010)

12. Niepert, M., Noessner, J., Stuckenschmidt, H.: Log-linear description logics. In: IJCAI. pp. 2153-2158 (2011)

13. Nilsson, N.J.: Probabilistic logic. Artificial Intelligence 28, 71-88 (February 1986)

14. Paris, J.B.: The Uncertain Reasoner's Companion - A Mathematical Perspective. Cambridge University Press (1994)

15. Peñaloza, R., Potyka, N.: Probabilistic reasoning in the description logic $\mathcal{A L C P}$ with the principle of maximum entropy. In: International Conference on Scalable Uncertainty Management. pp. 246-259. Springer (2016)

16. Peñaloza, R., Potyka, N.: Towards statistical reasoning in description logics over finite domains (full version). CoRR abs/1706.03207 (2017), http: / / arxiv.org/abs/1706. 03207

17. Potyka, N., Thimm, M.: Probabilistic reasoning with inconsistent beliefs using inconsistency measures. In: IJCAI. pp. 3156-3163 (2015)

18. Riguzzi, F., Bellodi, E., Lamma, E., Zese, R.: Probabilistic description logics under the distribution semantics. Semantic Web 6(5), 477-501 (2015) 